\title{
A test for psychometric function shift
}

\author{
Alexander D. Logvinenko • Yuri N. Tyurin • \\ Martin Sawey
}

Published online: 16 November 2011

(C) Psychonomic Society, Inc. 2011

\begin{abstract}
A nonparametric, small-sample-size test for the homogeneity of two psychometric functions against the left- and right-shift alternatives has been developed. The test is designed to determine whether it is safe to amalgamate psychometric functions obtained in different experimental sessions. The sum of the lower and upper $p$ values of the exact (conditional) Fisher test for several $2 \times 2$ contingency tables (one for each point of the psychometric function) is employed as the test statistic. The probability distribution of the statistic under the null (homogeneity) hypothesis is evaluated to obtain corresponding $p$-values. Power functions of the test have been computed by randomly generating samples from Weibull psychometric functions. The test is free of any assumptions about the shape of the psychometric function; it requires only that all observations are statistically independent.
\end{abstract}

Keywords Psychophysics · Psychometric function · Threshold

A. D. Logvinenko $(\bowtie)$

Department of Vision Sciences, Glasgow Caledonian University,

Glasgow G4 0BA, UK

e-mail: a.logvinenko@gcu.ac.uk

Y. N. Tyurin

Department of Probability Theory and Mathematical Statistics,

Moscow State University,

Moscow, Russia

M. Sawey

School of Psychology, Queen's University, Belfast BT9 5BP, UK

\section{Introduction}

Many of the widely used techniques for measuring threshold imply an evaluation of the so-called psychometric function, defined as the probability of obtaining one of two observer responses (e.g., yes or no in the classical yes-no experimental paradigm; or correct/incorrect in the twoalternative forced choice $[2 \mathrm{AFC}]$ experimental paradigm) depending on the intensity (or contrast) of a presented stimulus (for a review, see, e.g., Guilford, 1954; Klein, 2001; Wichmann \& Hill, 2001a, 2001b; Woodworth \& Schlosberg, 1955). As a rule of thumb, at least a few hundred observations per psychometric function are needed to estimate a threshold reasonably reliably. Although one such experiment may be completed in one sitting, a one hundred threshold measurement is usually the maximum of what can sensibly be done in one psychophysical experimental session. Furthermore, it is rare that just one threshold is to be determined. On the contrary, it is common practice for thresholds for many different stimuli to be determined at the same sitting. This is particularly the case when studying, for instance, the effect of stimulus dimensions, such as size, shape, spatial and temporal frequencies, duration, and so forth, on stimulus threshold. The number of thresholds to be measured in such an experiment is too large to be completed in one experimental session. In this case, an experiment is usually divided into separate sessions, the results from each session being combined afterward.

Since experimental sessions may be separated by days or even weeks, the data from different sessions should be tested for homogeneity before they are amalgamated. Although the need for such a test appears self-evident, there are at least two particular reasons for it that deserve to be mentioned. First, because of learning fatigue, observers 
may gradually change the response pattern over time; that is, they may exhibit nonstationary performance (Wichmann \& Hill, 2001a). Second, as many psychophysicists know, a sudden change in the observer's response patterns may occur from time to time. In particular, a shift may occur in the psychometric function along the stimulus axis from session to session, thus making the amalgamation of data from different sessions inappropriate. If the number of measurements is big enough, this shift in experimentally obtained psychometric functions can be spotted by the eye. ${ }^{1}$ However, this is not the case when the number of measurements per stimulus is small.

For example, the two psychometric functions in Fig. 1 were obtained from the same observer in two different experimental sessions, in which contrast thresholds for the sinusoidal gratings of $30 \mathrm{c} / \mathrm{deg}$ were being measured. Each grating contrast was presented 5 times during one session. ${ }^{2}$ Obviously, it is not easy to decide whether it is safe to amalgamate these two functions or not without a suitable statistical instrument. ${ }^{3}$ One, seemingly plausible, approach to making such a decision is to fit the data with some reasonable approximating function (e.g., a Weibull or a logistic function) and then to check whether or not the difference between the estimated parameters in question (i.e., threshold) across two experimental sessions significantly differs from zero. However, such an approach would hardly be appropriate when the observed frequencies (i.e., empirical estimates of the probability) constituting the experimental psychometric functions result from a number of presentations that is not large (say, not more than five, as in the example given above). As a rule, the true sample distributions of the estimated parameters are unknown, so testing the difference between them, as with any other statistical hypothesis about parameters, is best based on their asymptotic distributions. The substitution of a true (but unknown) distribution by an asymptotic one is acceptable only when the sample size is large, and that, as was mentioned above, is not the case we address here.

\footnotetext{
${ }^{1}$ Though, with the current trend for commercial computer hardware controlling the execution of psychophysical experiments, as well as an analysis of psychophysical data, it is not always possible to inspect interim results.

${ }^{2}$ In psychophysical practice, each stimulus is often presented even less than 5 times during one experimental session. For instance, given that the grating spatial frequency varied, say, at ten levels, simple arithmetic calculation shows that in one experiment session, one cannot present each grating more than 4 times (assuming that every psychometric function is sampled at ten points, at least), if the whole number of presentations during the session is not to exceed 400 .

${ }^{3}$ It should be noted that the irregular form of the psychometric functions is mainly due to the small number of observations. When the number of observations per point is sufficiently large-for example, more than 50 - the same (well-experienced in psychophysical experiments) observer would usually produce quite smooth and monotonic psychometric functions.
}

Over and above this, the empirical psychometric functions obtained from a small number of observations are often not monotonic, as can be seen in Fig. 1, so there is little point in fitting them with a monotonic function, such as a Weibull or a logistic. In particular, when we tried to estimate the two parameters of the Weibull function (see Eq. 12 below), using the maximum likelihood technique, either the standard algorithm did not converge, or the estimated parameters were not realistic. This is hardly surprising, since the main reason we would amalgamate psychometric functions from small experimental sessions is to get a data set large enough to obtain a reliable estimation of the parameters of an approximating function. In other words, testing for a shift is supposed to precede fitting, not the other way round. Besides, an approach based on prior fitting implies a shape for the psychometric function, whereas it is desirable to have a test free of such an assumption.

So, there is a need for a nonparametric technique to test the hypothesis that two psychometric functions obtained in different experimental sessions come from a homogeneous population, against the alternative hypothesis that they are shifted relative to each other. Furthermore, it should be a test that is safe to use when the number of repetitions per stimulus value is very small. Since no statistical test with such features seems to be available in the literature, in this article, we report our own developments on this subject. The test we present meets all the requirements above. In particular, it makes no assumption about the shape of the psychometric function, and it allows the number of stimulus presentations to be however small. The only restrictive assumption made is that of homogeneity within the experimental session.

\section{Theory}

Suppose that the probability of one of the two possible observer responses-yes $(\mathrm{Y})$, say ${ }^{4}$ - depends on a quantitative variable (e.g., intensity, or contrast, of stimulus) $z$, with

$P(Y \mid z)=p(z)$,

where $P(Y \mid z)$ stands for the probability of $Y$ given $z$. Suppose that the probability $p(z)$ remains constant for every stimulus presentation during a session but can differ from one session to another. Let us assume, then, that $m$ levels of the variable $z\left(z_{1}<z_{2}<\ldots<z_{i}<\ldots<z_{m}\right)$ were presented in two experimental sessions. Let $p_{1}^{(i)}$ and $p_{2}^{(i)}$ be the

\footnotetext{
${ }^{4}$ The theory is equally applicable to a $2 \mathrm{AFC}$ experimental design.
} 


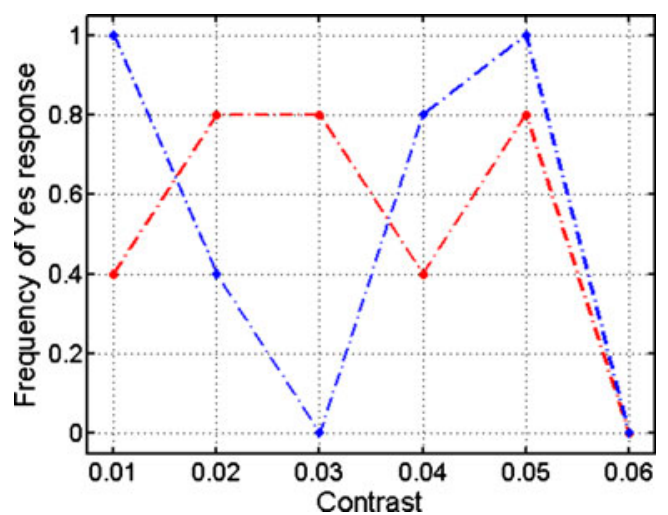

Fig. 1 Two psychometric functions from two separate experimental sessions. Five equally spaced levels of contrast (plotted on the horizontal axes) were used in each session. A stimulus of each contrast level was presented 5 times in a session. The number of yes responses divided by the whole number of presentations (i.e., five) is plotted on the vertical axes. The question is whether these two functions are from a homogeneous population. Put simply, can they be combined together?

probabilities of the yes response to stimulus value $z_{i}$ $(i=1, \ldots, m)$ in the first and second sessions, respectively.

A set of responses to the presentation of every stimulus value $z_{i}$ across two sessions can be condensed into a $2 \times 2$ table, written $T^{(i)}$ (see Table 1). In this table, $n_{11}^{(i)}$ and $n_{21}^{(i)}$ are the number of observed yes responses to stimulus value $z_{i}$ in the first and second sessions, respectively; $n_{12}^{(i)}$ and $n_{22}^{(i)}$ are the number of $n o$ responses to stimulus value $z_{i}$ in the first and second sessions, respectively. The subscript " + " denotes summation over the index it replaces; that is,

$n_{+1}^{(i)}=n_{11}^{(i)}+n_{21}^{(1)}$;

$n_{+2}^{(i)}=n_{12}^{(i)}+n_{22}^{(1)}$;

$n_{1+}^{(i)}=n_{11}^{(i)}+n_{12}^{(1)}$;

$n_{2+}^{(i)}=n_{21}^{(i)}+n_{22}^{(1)}$;

$n_{++}^{(i)}=n_{1+}^{(i)}+n_{2+}^{(i)}=n_{+1}^{(i)}+n_{+2}^{(i)}$ being the total sample size for the stimulus value $z_{i}$ in the two sessions.

Since the number of stimulus presentations in a psychophysical experiment is usually determined by the experimenter in advance, we will assume that two marginal frequencies - namely, the number of trials run in both sessions, $n_{1+}^{(i)}$ and $n_{2+}^{(i)}$-are fixed (not necessarily the same

Table 1 A $2 \times 2$ table associated with the stimulus $x$

\begin{tabular}{lccc}
\hline & Yes & No & Total \\
\hline Experimental session 1 & $n_{11}^{(i)}$ & $n_{12}^{(i)}$ & $n_{1+}^{(i)}$ \\
Experimental session 1 & $n_{21}^{(i)}$ & $n_{22}^{(i)}$ & $n_{2+}^{(i)}$ \\
Total & $n_{+1}^{(i)}$ & $n_{+2}^{(i)}$ & $n_{++}^{(i)}$ \\
\hline
\end{tabular}

numbers though). Hence, Table 1 is fully identified by one of its two columns (say, that associated with the yes response) - that is, by the frequencies $n_{11}^{(i)}$ and $n_{21}^{(i)}$. We will also assume that the observer's responses are mutually independent. In this case, the frequencies $n_{11}^{(i)}$ and $n_{21}^{(i)}$ can be considered as two independent binomial variables.

We wish to develop a statistic to determine whether the psychometric functions obtained in the two sessions may be considered to be empirical samples of the same theoretical psychometric functions. In other words, we wish to test the hypothesis

$H_{0}: p_{1}^{(1)}=p_{2}^{(1)}, \ldots, p_{1}^{(m)}=p_{2}^{(m)}$.

We assume that the psychometric functions from different sessions may be shifted relative to each other. This means that we test the hypothesis $H_{0}$ against the following alternatives, which, purely for convenience, we label positive and negative, respectively (Fig. 2):

$H_{+}: p_{1}^{(1)}>p_{2}^{(1)}, \ldots, p_{1}^{(m)}>p_{2}^{(m)}$ and $H_{-}: p_{1}^{(1)}<p_{2}^{(1)}, \ldots, p_{1}^{(m)}<p_{2}^{(m)}$.

The compound hypothesis $H_{0}$ is equivalent to the set of $m$ simple homogeneity hypotheses for each contingency table $T^{(i)}$. The rationale for the present study is to choose an appropriate measure of homogeneity for such a table and to sum up this measure of homogeneity across all the individual contingency tables $T^{(i)}$ in a single statistic.

Although there is a large volume of literature concerned with the problem of measuring and testing homogeneity in a single $2 \times 2$ table (for a review, see, e.g., Kroll, 1989; Mehta, 1994), it is not clear how to test homogeneity in

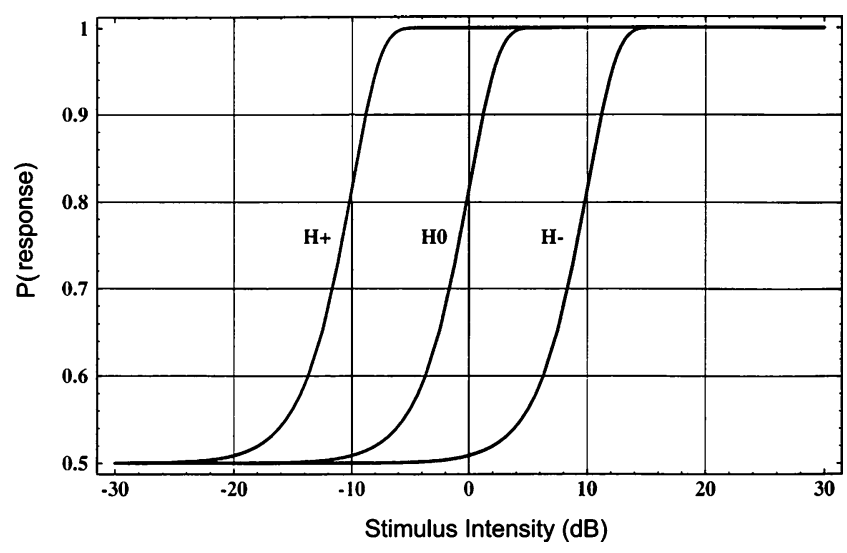

Fig. 2 Three psychometric functions are plotted according to Eq. 12 for three different values of the threshold parameter $t=-10 \mathrm{~dB}, 0 \mathrm{~dB}$, and $10 \mathrm{~dB}$ (from left to right). Probability of the correct response (vertical axis) as a function of intensity stimulus is expressed in decibels (horizontal axis).The leftmost and rightmost curves, together with the middle curve representing the reference psychometric function, illustrate the $H_{+}$and $H_{-}$alternatives, respectively 
several $2 \times 2$ contingency tables when the total sample size is small for every table. In this case, one cannot use Pearson and likelihood-ratio statistics, which are usually employed to test homogeneity null hypotheses for contingency tables (see, e.g., Agresti, 1984, p. 10; Kendall \& Stuart, 1979, pp. 577-580; Mehta, 1994). These statistics are known to have an asymptotic (as the total sample size $n_{++} \rightarrow \infty$ ) chisquared distribution. Since the number of presentations for each stimulus is expected to be small, all such asymptotic measures of homogeneity in a $2 \times 2$ table suitable for large samples cannot appropriately be used in our situation. Besides, the Pearson and likelihood-ratio tests are assumed to perform against all possible alternatives, whereas the task of revealing a shift in psychometric function statistically reduces to a test against one-sided alternatives. ${ }^{5}$

It seems more appropriate for our purpose to make use of the approach first proposed by Fisher when developing his exact test of homogeneity in a $2 \times 2$ table (e.g., Agresti, 1990, pp. 59-62; Kendall \& Stuart, 1979, pp. 580-582). The Fisher exact test is often referred to as conditional, since it implies an evaluation of the probability distribution of the cell frequencies in Table 1 conditioned by the marginal frequencies. Although there is some controversy concerning the adequacy of the Fisher exact test in the circumstances under consideration in this article (see the Discussion section), it is shown in Appendix 1 that it is appropriate for our purposes.

More specifically, in Appendix 1, it is shown that given a set of tables $X^{(i)}$ that have the same marginal frequencies as $T^{(i)}$,

$X^{(i)}=\left[\begin{array}{ll}x_{11}^{(i)} & x_{12}^{(i)} \\ x_{21}^{(i)} & x_{22}^{(i)}\end{array}\right]$,

where $x_{11}^{(i)}+x_{21}^{(i)}=n_{+1}^{(i)} ; x_{12}^{(i)}+x_{22}^{(i)}=n_{+2}^{(i)} ; x_{11}^{(i)}+x_{12}^{(i)}=n_{1+}^{(i)}$; $x_{21}^{(i)}+x_{22}^{(i)}=n_{2+}^{(i)}$; the conditional probability of observing the table $X^{(i)}$ on the null hypothesis is

$P\left(X^{(i)} \mid n_{+1}^{(i)}, n_{+2}^{(i)}\right)=\frac{\left(\begin{array}{c}n_{1+}^{(i)} \\ x_{11}^{(i)}\end{array}\right)\left(\begin{array}{c}n_{2+}^{(i)} \\ x_{21}^{(i)}\end{array}\right)}{\left(\begin{array}{c}n_{++}^{(i)} \\ n_{+1}^{(i)}\end{array}\right)}$.

Taking into account that when the marginal frequencies are fixed, the value of one cell in $X^{(i)}$ determines the other

\footnotetext{
${ }^{5}$ Although these tests may be improved so as to perform better against one-sided alternatives, and even to incorporate order information that is actually available in the alternative hypotheses $H_{+}$and $H_{-}$(e.g., Barlow, Bartholomew, Bremmer, \& Brunk, 1972; Robertson, 1988), we cannot follow this approach, since once again it is based on the chi-squared approximation and it would not be reliable for the small sample sizes for which our test is mainly required.
}

three, we arrive at the hypergeometrical distribution from which the Fisher exact test is computed:

$f^{(i)}\left(x_{11}^{(i)}\right)=\frac{\left(\begin{array}{c}n_{1+}^{(i)} \\ x_{11}^{(i)}\end{array}\right)\left(\begin{array}{c}n_{2+}^{(i)} \\ n_{+1}^{(i)}-x_{11}^{(i)}\end{array}\right)}{\left(\begin{array}{c}n_{++}^{(i)} \\ n_{+1}^{(i)}\end{array}\right)}$.

When the positive alternative hypothesis $H_{+}^{(i)}$ : $p_{1}^{(i)}>p_{2}^{(i)}, i=1, \ldots, m$, holds true, one becomes more likely to observe large values of $x_{11}^{(i)}$ and less likely to observe small ones. On the contrary, under $H_{-}$, the probability of observing large values of $x_{11}^{(i)}$ decreases, and that of observing small values increases. So under the alternative $H_{+}$for a given marginal total (2), the larger $n_{11}^{(i)}$, the stronger the evidence in favor of this alternative. As a quantitative measure of evidence against $H_{0}^{(i)}$ in favor of $H_{+}^{(i)}$, one can take the so-called $p$-value (let us designate it $v_{U}^{(i)}$, which is defined as the probability that the variable $x_{11}^{(i)}$ in Eq. 5 is at least as favorable to $H_{+}^{(i)}$ as the observed cell frequency $n_{11}^{(i)}$ in the table $T^{(i)}$. In order to evaluate the $p$-value for a given observed $n_{11}^{(i)}$, one has to sum the hypergeometric probabilities (Eq. 7) for all $x_{11}^{(i)}$ at least as large as the observed $n_{11}^{(i)}$. Since the range for $x_{11}^{(i)}$ in the hypergeometrical distribution is (Kendall \& Stuart, 1979, p. 581) $n_{\min }^{(i)}=$ $\max \left(0, n_{1+}^{(i)}+n_{+1}^{(i)}-n_{++}^{(i)}\right) \leq x_{11}^{(i)} \leq n_{\max }^{(i)}=\min \left(n_{1+}^{(i)}, n_{+1}^{(i)}\right)$, we have

$v_{U}^{(i)}=\sum_{n_{11}^{(i)} \leq \alpha \leq n_{\max }^{(i)}} f^{(i)}(\alpha)$

Note that the summation in Eq. 8 is taken over all integers $\alpha$ within the limits shown.

Considering each $p$-value $v_{U}^{(i)}$ as a random variable, the probability distribution of $v_{U}^{(i)}$ under the null hypothesis $H_{0}^{(i)}$ is specified by a table, referred to as $A_{U}^{(i)}$ (Table 2), where $a=n_{\min }^{(i)}$ and $b=n_{\max }^{(i)}$. Under the hypothesis $H_{0}^{(i)}, v_{U}^{(i)}$ is "roughly" uniformly distributed. The graph of its cumulative distribution function, $y=P\left(v_{U}^{(i)} \leq x\right)$, where $0 \leq x \leq 1$, has a staircase form (Fig. 3). The greater the number of points in the distribution, the narrower the width of the staircase and, hence, the closer the distribution to a straight line.

On the alternative $H_{+}^{(i)}$, the probability distribution of $v_{U}^{(i)}$ is different. The probability of a small $v_{U}^{(i)}$ increases, and that of a large $v_{U}^{(i)}$ decreases, as compared with those under the null hypothesis $H_{0}^{(i)}$. Therefore, the smaller the $p$-value 
Table 2 The probability distribution of $v_{U}^{(i)}\left(A_{U}^{(i)}\right)$

\begin{tabular}{lllll}
\hline$p$ - values & $v_{U}^{(i)}(a)$ & $v_{U}^{(i)}(a+1)$ & $\ldots$ & $v_{U}^{(i)}(b)$ \\
\hline Probabilities & $f^{(i)}(a)$ & $f^{(i)}(a+1)$ & $\ldots$ & $f^{(i)}(b)$ \\
\hline
\end{tabular}

(i.e., the probability of rejecting the null hypothesis when it is true), the stronger the evidence against $H_{0}^{(i)}$ and in favor of $H_{+}^{(i)}$ at stimulus value $x_{i}$.

Likewise, a small cell frequency $n_{11}^{(i)}$ for given marginal values points to the negative hypothesis $H_{-}^{(i)}: p_{1}^{(i)}<p_{2}^{(i)}$. The corresponding $p$-value (written $v_{L}^{(i)}$ ) - that is, the sum of hypergeometric probabilities for outcomes at least as small as the observed value-is

$v_{L}^{(i)}=\sum_{n_{\min }^{(i)} \leq \alpha \leq n_{11}^{(i)}} f^{(i)}(\alpha)$.

Correspondingly, the probability distribution of $v_{L}^{(i)}$ is given by the analogous table designated $A_{L}^{(i)}$ (Table 3 ). If close to zero, $v_{L}^{(i)}$ testifies against $H_{0}^{(i)}$ and in favor of $H_{-}^{(i)}$.

Turning to the null hypothesis (Eq. 2) to test the homogeneity of entire psychometric functions, we have to decide how to combine the individual $p$-values considered as a measure of homogeneity for individual tables $T^{(i)}$ into a global homogeneity test. While it has been discussed how to combine $p$-values when the test statistic has a continuous distribution and, thus, $p$-values are uniformly distributed over the interval [0;1] (e.g., Rosenthal, 1993), there seems to exist no technique for combining $p$-values from separate Fisher's exact tests when the $p$-values have essentially discrete distributions. At any rate, we know of only one work where a similar problem has been considered (Zelen, 1971). More specifically, Zelen has developed a nonparametric statistic to test the null hypothesis, expressed in terms of odds ratios, which is quite similar to our $H_{0}$. However, his statistic was designed to test $H_{0}$ against all the alternatives, whereas we wish to test it against one-sided (i.e., shift) alternatives (Eq. 4). There is also another essential difference between his null hypothesis and ours that prevents us from following his approach. ${ }^{6}$

${ }^{6}$ Zelen (1971) considered the hypothesis that the odds ratios for all $\mathrm{m}$ $2 \times 2$ tables are the same:

$\Psi_{1}=\Psi_{2}=\ldots=\Psi_{m}(=\Psi$, say $)$,

where $\Psi_{i}=\frac{p_{1}^{(i)}}{1-p_{1}^{i}}: \frac{p_{2}^{(i)}}{1-p_{2}^{i}}, i=1, \ldots, m$. The generic set he considered consisted of $m$ tables with the same marginal frequencies as the observed tables $T^{(i)}(i=1, \ldots, m)$ and an additional restriction that the sum $x_{11}^{(1)}+x_{11}^{(2)}+\ldots+x_{11}^{(m)}$ was the same as observed. The null hypothesis we consider here (Eq. 2) is slightly different-namely, $\Psi_{1}=\Psi_{2}=\ldots=\Psi_{m}=1$.

In fact, the generic set for the hypothesis $\left({ }^{* *}\right)$ is broader than that for the hypothesis $(*)$, and it is this that makes a difference between our statistic and Zelen's.

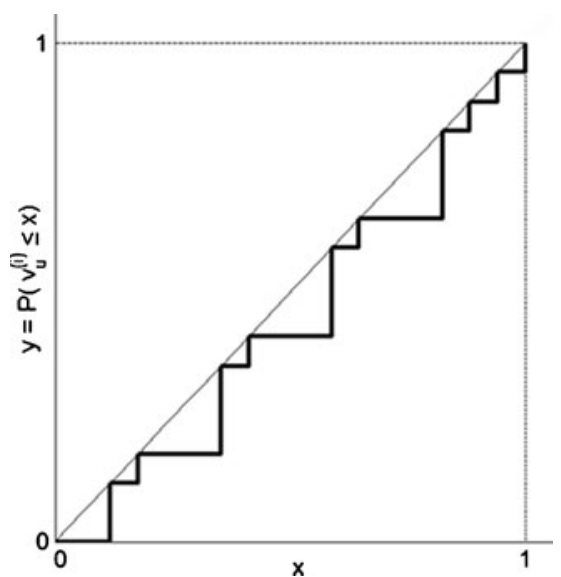

Fig. 3 Graph of the cumulative distribution function of $v_{U}^{(i)}$ under the null hypothesis $H_{0}^{(i)}$

So we have decided simply to sum the individual $p$-values and then to evaluate the exact probability distribution of the obtained sums by direct calculation. Specifically, we take the sum of upper individual $p$-values,

$v_{U}=\sum_{i=1}^{m} v_{U}^{(i)}$

as a statistic to test the null hypothesis $H_{0}$ against $H_{+}$. The sum of lower individual $p$-values,

$v_{L}=\sum_{i=1}^{m} v_{L}^{(i)}$

will be used to test the null hypothesis $H_{0}$ against $H_{-}$. (Recall that $m$ in [10] and [11] is the number of points in the psychometric function.)

Indeed, all the individual $p$-values have the same limits of variation. Under the null hypothesis $H_{0}$ they are all "nearly" uniformly distributed. Under the alternatives $H_{+}$ (respectively, $H_{-}$), all the values $v_{U}^{(i)}(i=1, \ldots, m)$ (respectively, $\left.v_{L}^{(i)}\right)$ tends toward zero. Intuitively, it seems natural to expect that this tendency cannot but become stronger if we sum up all the upper (respectively, lower) $p$-values in a single index $v_{U}$ (respectively, $v_{L}$ ).

The extent to which the observable data provide evidence against $H_{0}$ in favor of $H_{+}$(respectively, $H_{-}$) can be evaluated in terms of the probability that when $H_{0}$ holds true the value $v_{U}$ (respectively, $v_{L}$ ) is more (respectively,

Table 3 The probability distribution of $v_{L}^{(i)}\left(A_{L}^{(i)}\right)$

\begin{tabular}{lllll}
\hline$p$ - values & $v_{L}^{(i)}(a)$ & $v_{L}^{(i)}(a+1)$ & $\cdots$ & $v_{L}^{(i)}(b)$ \\
\hline Probabilities & $f^{(i)}(a)$ & $f^{(i)}(a+1)$ & $\ldots$ & $f^{(i)}(b)$ \\
\hline
\end{tabular}


less) than or equal to that observed. We reject the null hypothesis $H_{0}$ in favor of $H_{+}$(respectively, $H_{-}$) if the $p$-value of the observed combined value $v_{U}$ (respectively, $v_{L}$ ) is small enough. For these $p$-values to be calculated, one needs to evaluate the probability distributions of $v_{U}$ and $v_{L}$.

Under the null hypothesis, the distribution of $v_{U}$ (respectively, $v_{L}$ ) is the convolution of $\mathrm{m}$ discrete distributions for individual $p$-values, $v_{U}^{(i)}$ (respectively, $\left.v_{L}^{(i)}\right)(1 \leq i$ $\leq m$ ), since all the observations for various stimuli are assumed independent. A method to compute the $p$-values of $v_{U}$ (written $P_{U}$ ) and of $v_{L}$ (written $P_{L}$ ) by using a discrete convolution procedure is described in Appendix 2. If one of these numbers is close to zero, there is strong evidence against the null hypothesis $H_{0}$ in favor of the positive or negative alternatives, $H_{+}$or $H_{-}$, depending on which of the numbers $P_{U}$ or $P_{L}$, respectively, falls close to zero. More specifically, given a level of significance $\alpha$, the null hypothesis $H_{0}$ will be rejected in favor of $H_{+}$or $H_{-}$if either $P_{U}<\alpha$ or $P_{L}<\alpha$, respectively. ${ }^{7}$

\section{Computer simulation and power of the test}

In order to validate the test, we have simulated thresholdmeasuring experiments using the Weibull function, which has been employed by many investigators to model their observer's psychometric functions (e.g., Nachmias, 1981). To be more exact, to simulate a series of observer correct responses (written CR) to a stimulus with intensity $z_{i}$ in decibels, we used a binomial distribution with parameters $k$ (the number of presentations, which is kept constant across stimuli $\left.z_{i}\right)$ and $p_{i}(i=1, \ldots, m)$, the latter being described by the following function:

$p_{i}=P\left(C R \mid z_{i}\right)=1-0.5 \exp \left[-10^{\left(z_{i}-\tau\right) \frac{\beta}{20}}\right]$,

where $\tau$ is the threshold (in decibels), and the parameter $\beta$ determines the slope of the psychometric function (see Fig. 2).

We have simulated four different experiments corresponding to four combinations of the parameters $k$ and $m$ (recall that $m$ is the number of points in the psychometric function; $k$ is the number of observations for each $m$ ) namely, $m=k=5 ; m=5, k=10 ; m=10, k=5$; and $m=5$, $k=20$. The two sets of the stimulus values $z_{i}$ and the corresponding probabilities $P_{i}$ resulting from Eq. 12, are depicted in Figs. $4 \mathrm{a}(m=5)$ and $4 \mathrm{~b}(m=10)$ given $\tau=0$

\footnotetext{
${ }^{7}$ As Example 3 below shows, it may happen, however rarely, that while the null hypothesis is rejected, one cannot distinguish between the alternatives. However, such an unusual case may occur only when the level of significance is quite high (specifically, it was 0.25 in Example 3).
}

and $\beta=3.3$. Then, to study whether the range at which points are sampled from the Weibull function has any effect, we repeated the four simulations, using the wider range shown in Figs. $4 \mathrm{c}(m=5)$ and $4 \mathrm{~d}(m=10)$.

In the simulations, 10,000 pairs of psychometric functions were generated for each of the four combinations of parameters $m$ and $k$. The threshold and slope parameters used to generate the functions in a pair either were the same or differed in either $\tau$ or $\beta$ only (not both). More specifically, while the threshold parameter $\tau$ was varied from 0 to $40 \mathrm{~dB}$, the slope parameter $\beta$ was kept constant (equal to 3.5). When the slope parameter $\beta$, varied from 1 to $6, \tau$ was kept at 0 . Each pair generated was then tested for homogeneity by the calculation of $p$-values in the manner described in the previous section and Appendix 2 (Eq. 19).

When the null hypothesis is true, the $p$-values $P_{U}$ and $P_{L}$ have distributions close to uniform. Therefore, the cumulative distribution functions for the $p$-values $P_{U}$ and $P_{L}$ obtained under simulation corresponding to the situation when there is no change in threshold should be approximately a straight line through the origin and the point $(1,1)$. (For further details, see Appendix 3.) As can be seen in Fig. 5 (filled circles), the results for the simulations are in good agreement with this prediction.

When a nonzero difference in $\tau$ is introduced ( $\beta$ is the same and equal to 3.5), one of the two $p$-values, either $P_{U}$ or $P_{L}$ depending on the sign of the difference, tends to gravitate to zero, the other to one. As a result, the cumulative distribution function for the $p$-value that tends to zero is convex above the diagonal, with end points lying approximately on the diagonal itself (Fig. 5), while the $p$-value that tends to one is concave below the diagonal. The cumulative distribution functions for the lower $p$-value, $P_{L}$, evaluated for $\tau$ from 0 to $10 \mathrm{~dB}(\beta=3.5, m=k=5)$ are depicted in Fig. 5. In line with our prediction, they are convex above the diagonal.

Then, given a level of significance $\alpha$-that is, the probability of incorrectly rejecting the homogeneity hypothesis, $H_{0}$, when it is true - note that a point with abscissa $\alpha$ lying on one of the curves (say, corresponding to a threshold difference $t$ in Fig. 5) gives us the probability of rejecting $H_{0}$ when the alternative for this particular $t$ is true. This probability is usually called the power of the test against the alternative $t$. Plotted as a function of $t$, this probability is what is known statistically as the power function of the test.

Depicted in Fig. 6a are the power functions evaluated at various levels of significance (see the figure caption) for settings that simulate an experiment in which five points were tested along each psychometric function and five observations were made at each point (i.e., $m=5$ and $k=5$ ). ${ }^{8}$ As was

\footnotetext{
$\overline{{ }^{8} \text { It means that }}$ both values of $n_{1+}^{(i)}$ and $n_{2+}^{(i)}$ were equal to 5 , and the index $i$ in Eqs. 10 and 11 ran from 1 to 5, so that five values of $v_{L}^{(i)}$ and five values of $v_{U}^{(i)}$ were summed up, respectively.
} 
Fig. 4 Weibull psychometric functions (see Eq. 12) sampled at five points (a and $\mathbf{c}$ ) and 10 points (b and $\mathbf{d}$ ) for use in computer simulations. Plates c and $\mathbf{d}$ represent the wider range discussed in the text
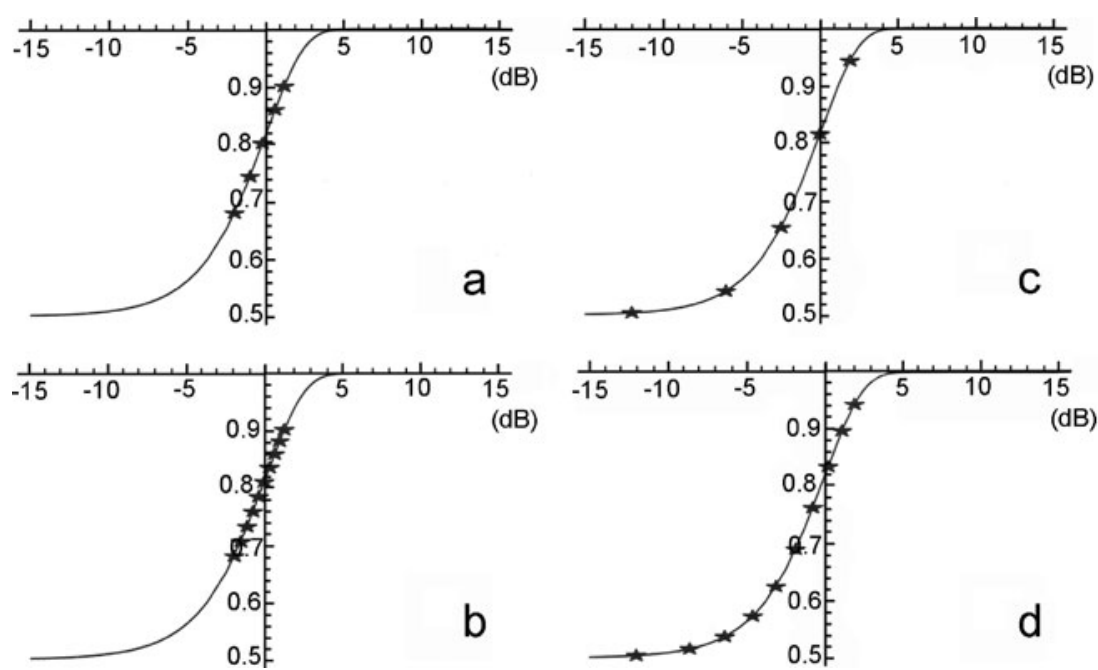

mentioned above, there were 10,000 comparisons of a pair of psychometric functions for each value of shift $t$ from 0 to 40 $\mathrm{dB}$. The power of the functions obtained for the other three combinations of parameters $m$ and $k$ are shown in Fig. 6b $(m=5 ; k=10)$, Fig. $6 \mathrm{c}(m=10 ; k=5)$, and Fig. $6 \mathrm{~d}(m=5$; $k=20)$.

The resemblance of the power functions in Fig. 6b and $\mathrm{c}$ shows that the power of the test depends on the whole number of presentations - that is, on the product "the number of stimulus values" times "the number of presentations of each stimulus value"-rather than on each factor separately. It means that one can achieve the

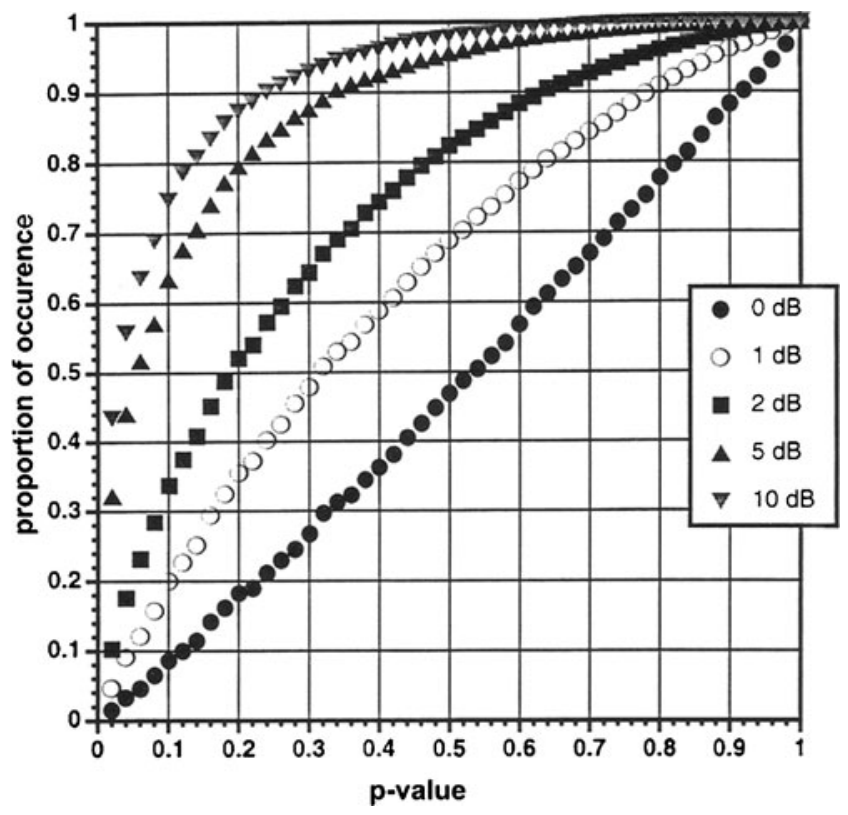

Fig. 5 The cumulative distribution functions for $p$-values (plotted along the horizontal axis) corresponding to different threshold shifts. An ordinate is the proportion of times that a $p$-value not more than a given particular abscissa was recorded during the simulation. Each curve corresponds to one particular difference in $\tau$ (shown in the legend) same power by increasing either the number of stimulus values or the number of presentations of each stimulus value.

It follows from Fig. 6 that the power of the test increases correspondingly with the whole number of presentations, but not as drastically as might be expected. Then, a comparison of the plots in Figs. 6 and 7 (narrow- and wide-sampled ranges, respectively) shows that the power of the test is better for the narrow range of the stimulus samples. This is hardly surprising, since the narrow-range samples contain more statistical information than do the wide-range samples. It should be noted, however, that it has been shown that wider sampling improves the estimation accuracy of the parameters of the psychometric function (Wichmann \& Hill, 2001b). Therefore, one has to trade off estimation accuracy versus the power to detect inhomogeneity (nonstationarity) in psychophysical data.

On the other hand, introducing a nonzero difference in $\beta$ ( $\tau$ 's the same) has no effect on the shape of the cumulative distribution function (Fig. 8). This shows that the test is quite robust relative to fluctuations of $\beta$, at least within limits typical for psychophysical experiments. This guarantees that, using this test, one will not mistake a change in $\beta$ for a shift in sensitivity.

\section{Examples}

To give an idea of how our test works in practice, the following examples are presented.

\section{Example 1}

Here, we test whether two 5-point samples taken from Weibull psychometric functions (Eq. 12) shifted relative to each other by $10 \mathrm{~dB}$ (see Fig. 2) are homogeneous or not. The tested samples (i.e., simulations of the empirical psychometric 
Fig. 6 The power functions of the test for different significance levels $(\alpha=0.001,0.01,0.05$, $0.10,0.20$ and 0.25 ) for the narrow sampled range. The power is plotted as ordinate against threshold shift in decibels as abscissa. The plots of power functions go with significance level in ascending order - that is, the lowest plot corresponds to $\alpha=0.001$, the highest to $\alpha=0.25$ (see the discussion in text). The $m$ and $k$ values shown for $\mathbf{a}, \mathbf{b}, \mathbf{c}$, and $\mathbf{d}$ represent the number of sampled points and number of repetitions per point, respectively (a) $m=5 k=5$

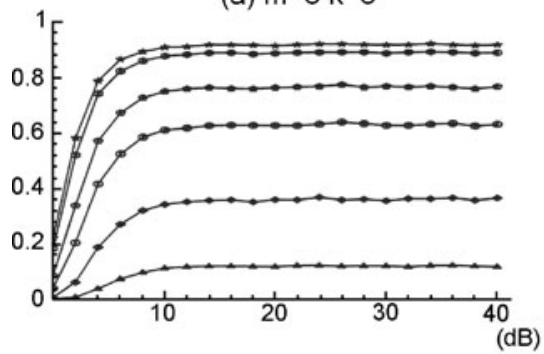

(b) $m=5 k=10$

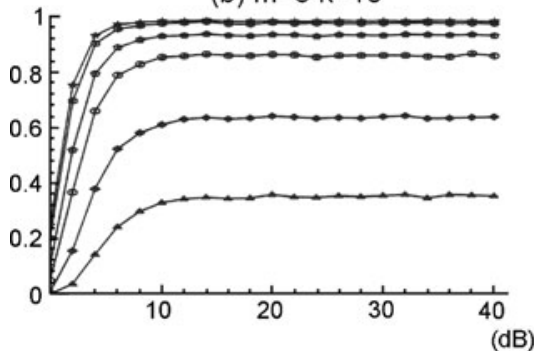

(c) $m=10 k=5$

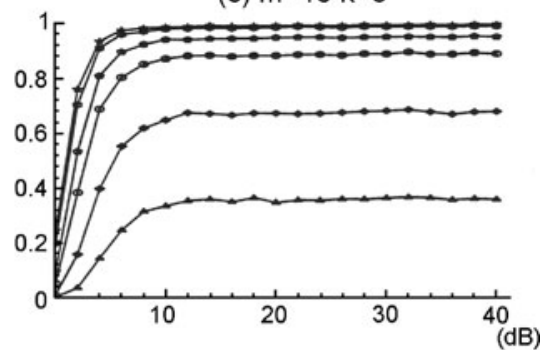

(d) $m=5 k=20$

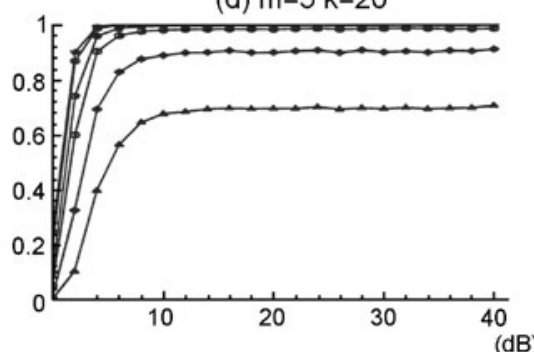

functions) are presented in Table $4(t=0 \mathrm{~dB}$ and $\beta=3.5$ in Eq. 12) and Table $5(t=10 \mathrm{~dB}$ and $\beta=3.5$ in Eq. 12).

The first step is to create a $2 \times 2$ table for each of the five pairs of stimuli (each pair consists of an observer's responses to one of the five stimuli from function 1 and the corresponding responses to the equivalent stimulus from function 2). For stimulus 1, we see from Table 4 that function 1 has two yes and 3 no responses and from Table 5 that function 2 has zero yes and five no responses. This leads to Table 6 (cf. Table 1).

The next step is to evaluate the lower and upper $p$-values (Eqs. 8 and 9, respectively). For the sake of brevity, we will show only the evaluation of the lower $p$-value $v_{L}^{1}$ for stimulus 1 at some length, the other lower and upper $p$ values being evaluated the same way.
From Eq. 9, we see that $v_{L}^{1}$ is a summation of some values of the function $f^{(i)}$ in Eq. 7, each of which can be expressed purely in terms of the binomial coefficients of values obtained from Table 6 . For stimulus 1, the range for the distribution (7) (from $a=n_{\min }^{(1)}$ up to $b=n_{\max }^{(1)}$ ), is evaluated as the following:

$$
\begin{aligned}
a & =n_{\min }^{(1)}=\max \left(0, n_{1+}^{(1)}+n_{+1}^{(1)}-n_{++}^{(1)}\right) \\
& =\max (0,5+2-10)=\max (0,-3)=0 ; \\
b & =n_{\max }^{(1)}=\min \left(n_{1+}^{(1)}, n_{+1}^{(1)}\right)=2 .
\end{aligned}
$$

So we need to evaluate the values of the hypergeometric distributions $f^{(1)}\left(x_{11}^{(1)}\right)$ at $x_{11}^{(1)}=0,1$, and 2 . Remember
Fig. 7 The power functions of the test for different significance levels $(\alpha=0.001,0.01,0.05$, $0.10,0.20$ and 0.25 ) for the wider sampled range (cf. Fig. 4)

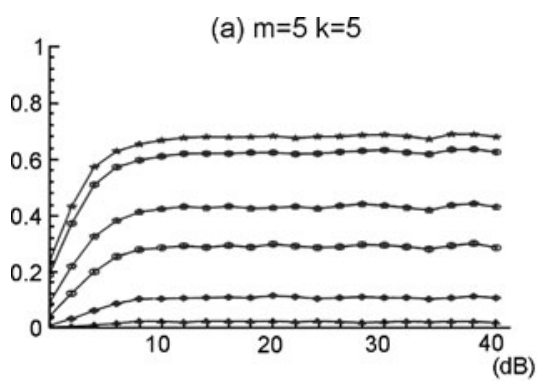

(b) $m=5 k=10$
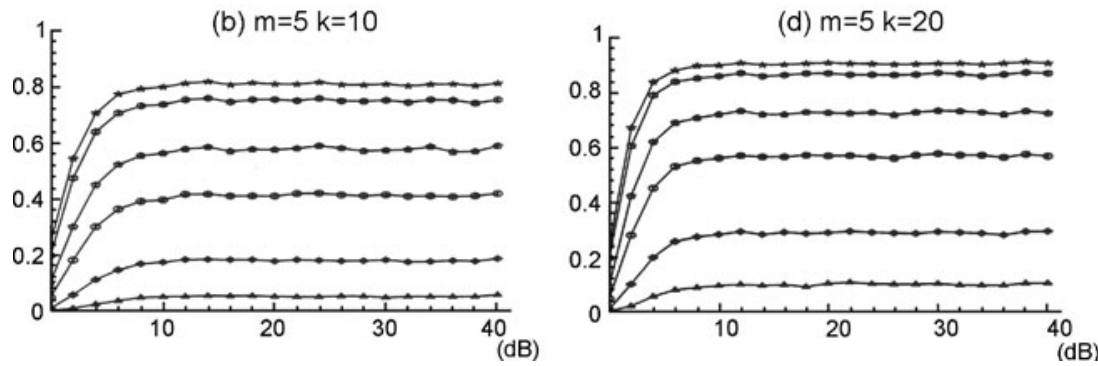


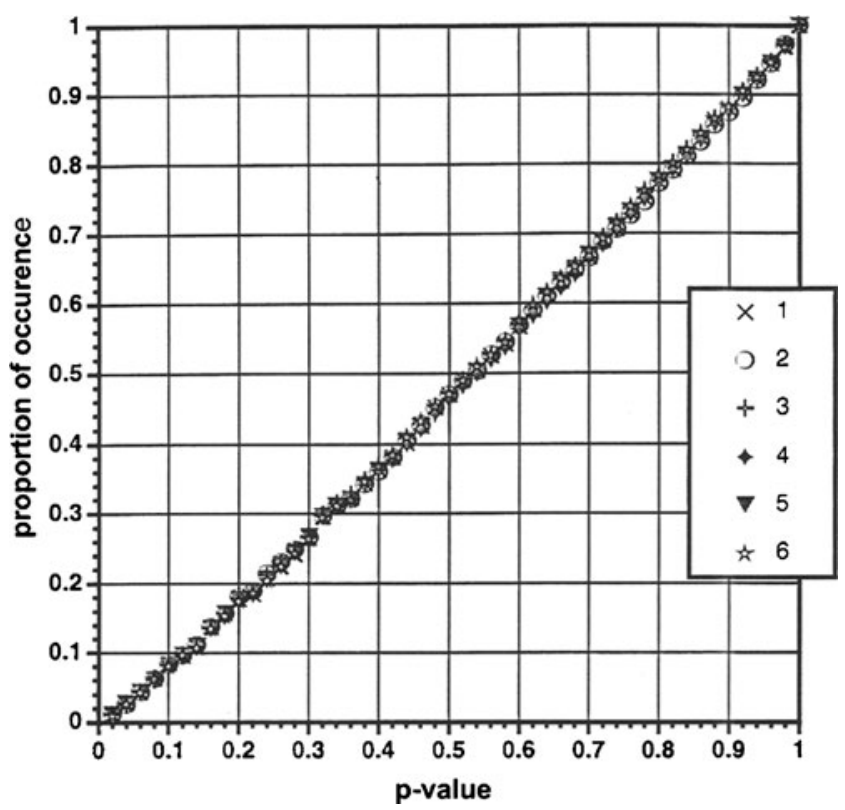

Fig. 8 The cumulative distribution functions for $p$-values corresponding to various different values of the parameter $\beta$. The axes are as in Fig. 5. In the legend are shown the values of $\beta$ used for the second psychometric function ( $\beta$ for the first one was always equal to 3.5$)$. The six separate plots are practically indistinguishable due to overlapping data, indicating that varying $\beta$ has no effect on the distribution function

that these values correspond to the probability of obtaining a particular $2 \times 2$ table with given marginal frequencies and the cell $n_{11}^{(1)}$ having the values 0,1 , and 2 , respectively. Using values from Table 6, we obtain

$f^{(1)}(0)=\frac{\left(\begin{array}{c}n_{1+}^{(1)} \\ 0\end{array}\right)\left(\begin{array}{c}n_{2+}^{(1)} \\ n_{+1}^{(1)}-0\end{array}\right)}{\left(\begin{array}{l}n_{++}^{(1)} \\ n_{+1}^{(1)}\end{array}\right)}=\frac{\left(\begin{array}{c}5 \\ 0\end{array}\right)\left(\begin{array}{c}5 \\ 2-0\end{array}\right)}{\left(\begin{array}{c}10 \\ 2\end{array}\right)}=0.222$.

Similarly, $f^{(1)}(1)$ is calculated to be 0.556 and $f^{(1)}(2)=0.222$. Adding up the obtained values, we evaluate the lower $p$-value for the first stimulus:

$v_{L}^{(1)}=f^{(1)}(0)+f^{(1)}(1)+f^{(1)}(2)=0.222+0.556+0.222=1$.

Table 4 Psychometric function 1 in Example 1

\begin{tabular}{llllll}
\hline Stimulus number & 1 & 2 & 3 & 4 & 5 \\
\hline Stimulus contrast (dB) & -12.0 & -6.0 & -2.5 & 0 & 1.9 \\
Number of Yes responses & 2 & 2 & 3 & 5 & 5 \\
Number of No responses & 3 & 3 & 2 & 0 & 0 \\
Total number of presentations & 5 & 5 & 5 & 5 & 5 \\
\hline
\end{tabular}

Table 5 Psychometric function 2 in Example 1

\begin{tabular}{llllll}
\hline Stimulus Number & 1 & 2 & 3 & 4 & 5 \\
\hline Stimulus contrast (dB) & -12.0 & -6.0 & -2.5 & 0 & 1.9 \\
Number of Yes responses & 0 & 4 & 2 & 2 & 1 \\
Number of No responses & 5 & 1 & 3 & 3 & 4 \\
Total number of presentations & 5 & 5 & 5 & 5 & 5 \\
\hline
\end{tabular}

Calculating the other four lower $p$-values in the same way as $v_{L}^{(1)}$, we get

$v_{L}^{(2)}=0.26, v_{L}^{(3)}=0.90, v_{L}^{(4)}=1.00$, and $v_{L}^{(5)}=1.00$.

From Eq. 11, the overall $v_{L}$ is simply the sum of the individual $v_{L}^{(i)}(i=1, \ldots, 5): \quad v_{L}=1.00+0.26+0.90+$ $1.00+1.00=4.16$. We now use this value with the convoluted distribution to obtain our final $p$-value: $P_{L}$. Simply, all values not more than $v_{L}$ in the distribution sum toward $P_{L}$ (Eq. 19 in Appendix 2). Doing the relevant sums gives us $P_{L}=0.8638$. Similarly, the upper $p$-value from Eq. 19 can be shown to be $P_{U}=0.0086$.

We see that the upper $p$-value is very close to zero. So we conclude that for this pair of psychometric functions, function 1 is significantly left-shifted relative to function 2 and, thus, the null hypothesis must be rejected in favor of $H_{+}$. Note that such a decision is quite clear because functions 1 and 2 were sampled from substantially different Weibull populations.

\section{Example 2}

In this example, we illustrate the case where there is strong evidence to reject the null hypothesis when it is true. Namely, we tested two psychometric functions that were samples of the same Weibull function $(t=0 \mathrm{~dB}$ and $\beta=$ 3.5). These functions are shown in Tables 7 and 8. We restrict ourselves to presenting final results. The $p$-values obtained for the resultant statistics $v_{L}$ and $v_{U}$ are $P_{L}=$ 0.0086 and $P_{U}=0.8313$. Since the lower $p$-value is close to zero, we have to reject the null hypothesis, although it is true, in favor of the alternative $H_{-}$(to be more exact, $H_{0}$ is rejected given a significance level $\alpha \leq 0.01$ ). This example illustrates how empirical psychometric functions may look when Type I error is made.

Table 6 The $2 \times 2$ table associated with stimulus 1 in Example 1

\begin{tabular}{llll}
\hline & Yes & No & Total \\
\hline Experimental session 1 & 2 & 3 & 5 \\
Experimental session 2 & 0 & 5 & 5 \\
Total & 2 & 8 & 10 \\
\hline
\end{tabular}


Table 7 Psychometric function 1 in Example 2

\begin{tabular}{llllll}
\hline Stimulus Number & 1 & 2 & 3 & 4 & 5 \\
\hline Stimulus contrast (dB) & -12.0 & -6.0 & -2.5 & 0 & 1.9 \\
Number of Yes responses & 1 & 0 & 4 & 3 & 5 \\
Number of No responses & 4 & 5 & 1 & 2 & 0 \\
Total number of presentations & 5 & 5 & 5 & 5 & 5 \\
\hline
\end{tabular}

\section{Example 3}

With this example (Tables 9 and 10), we illustrate the case where choosing the significance level as high as .25, as will be recommended below to reduce Type II error, leads us to refuse the null hypothesis but does not allow us to distinguish between the alternatives $H_{+}$and $H_{-}$.

The $p$-values for $v_{L}$ and $v_{U}$ were evaluated as $P_{L}=$ 0.2211 and $P_{U}=0.1930$. Therefore, given a significance level $\alpha \leq 0.25$, we have to refuse the null hypothesis. However, since both $p$-values are less than the significance level, we are unable to decide in favor of either of the alternatives $H_{-}$, or $H_{+}$.

\section{Discussion}

The statistical features of $2 \times 2$ contingency tables, on which the homogeneity test developed by us is based, are well established (e.g., Everitt, 1992; Kendall \& Stuart, 1979, Chap. 33). However, the problem of testing homogeneity in such tables is still controversial (for a review, see, e.g., Kroll, 1989). Particularly, the appropriateness and adequacy of using the Fisher exact test for testing two observed frequencies in the experimental circumstances we consider, remains unclear. ${ }^{9}$ For instance, the Fisher exact test has been criticized on the grounds that it is too conservative, and indeed, sometimes its size may prove to be even less than an assigned level of significance. This anomalous behavior is more likely to manifest itself for small samples. To remedy this, Haber (1990) has suggested using an alternative statistic. However, this cannot be used in our situation, because there is no obvious way to generalize this approach to the case of several separate $2 \times 2$ tables.

On the other hand, there seems to be no need to do this, because while both our test and Fisher's involve the conditional hypergeometric distribution, we make use of it in a rather different way. Fisher's test is directly based on

\footnotetext{
${ }^{9}$ A large literature is associated with this problem. Among those who have claimed in favor of the Fisher exact test, one can mention, particularly, Camilli (1990), Agresti (1984), Everitt (1993), and Mehta (1994). An alternative view can be found in Berkson (1978), Haber (1986, 1990), and Kroll (1989).
}

Table 8 Psychometric function 2 in Example 2

\begin{tabular}{llllll}
\hline Stimulus Number & 1 & 2 & 3 & 4 & 5 \\
\hline Stimulus contrast (dB) & -12.0 & -6.0 & -2.5 & 0 & 1.9 \\
Number of Yes responses & 4 & 3 & 2 & 5 & 5 \\
Number of No responses & 1 & 2 & 3 & 0 & 0 \\
Total number of presentations & 5 & 5 & 5 & 5 & 5 \\
\hline
\end{tabular}

the distribution (7) associated with a single $2 \times 2$ table, whereas ours is based on a statistic that is a convolution of random variables emerging from several $2 \times 2$ tables. When the samples are small, the size of the Fisher exact test may differ considerably from the assigned level of significance $\alpha$. Although the conditional distribution on which our test is based is also discrete, the length of a distribution that results from convolution is greater than the originals, so for this reason alone, we would not expect our test to be as conservative as the Fisher exact test. Nevertheless, we decided to evaluate directly the size of our test.

Specifically, for different levels of $\alpha$ at which $H_{0}$ is formally rejected (the nominal level of significance), we have computed the relative frequencies of false rejections of the null hypothesis, $H_{0}$, (the effective $\alpha$ ) for different values of parameters $k$ and $m$. The results are shown in Table 11 . While there are some differences between the nominal and effective $\alpha$, the power of our test is very close to its size. In particular, it seems that the difference between them for the whole number of presentations $m \times k \geq 50$ could be put down to random fluctuations unavoidable under computer simulation. At any rate, we conclude that the power of our test is quite appropriate for the circumstances typical for psychophysical experiments.

It should be mentioned that while choosing a nominal level of significance, one needs to keep in mind that different attitudes might be adopted toward errors of the first and second types. Indeed, when testing homogeneity, to refuse the null hypothesis when it is true (Type I error) is usually more tolerable than to accept it when it is not true (Type II error). A consequence of the former is the repetition of an experiment the outcome of which was erroneously thrown away, whereas a consequence of the latter is corruption of the entire experimental data set obtained up to that point. However, when gathering data is

Table 9 Psychometric function 1 in Example 3

\begin{tabular}{llllll}
\hline Stimulus number & 1 & 2 & 3 & 4 & 5 \\
\hline Stimulus contrast (dB) & -12.0 & -6.0 & -2.5 & 0 & 1.9 \\
Number of Yes responses & 0 & 3 & 2 & 5 & 5 \\
Number of No responses & 5 & 2 & 3 & 0 & 0 \\
Total number of presentations & 5 & 5 & 5 & 5 & 5 \\
\hline
\end{tabular}


Table 10 Psychometric function 2 in Example 3

\begin{tabular}{llllll}
\hline Stimulus number & 1 & 2 & 3 & 4 & 5 \\
\hline Stimulus contrast (dB) & -12.0 & -6.0 & -2.5 & 0 & 1.9 \\
Number of Yes responses & 3 & 0 & 4 & 3 & 5 \\
Number of No responses & 2 & 5 & 1 & 2 & 0 \\
Total number of presentations & 5 & 5 & 5 & 5 & 5 \\
\hline
\end{tabular}

costly (e.g., in clinical studies), a different attitude might be more appropriate.

Another practical consideration that should be taken into account in specifying a significance level is that it is very unlikely that a threshold will be absolutely stable during an experimental period. There are unavoidable anatomophysiological sources of sensitivity fluctuation that may be tolerated, provided they fall within some predetermined limits, not more than say $t_{0}$.

Therefore, it would be good practice to set a level of significance so that the power of the test against the alternatives exceeding $t_{0}$ is not less than some conventional value. For instance, if the significance level is equal to .25 , then as we can see from Table 12, the power of the test against the alternative $t>t_{0}=2 \mathrm{~dB}$ is not less than .58 , provided that the whole number of presentations is not less than $25(m=5 ; k=5)$. It means that the null hypothesis will be rejected with a $25 \%$ risk of the psychometric functions not being amalgamated when they safely can be and a $42 \%$ risk of them being amalgamated when they are in reality shifted relative to each other by at least $2 \mathrm{~dB}$ (thus corrupting the data set). If a further reduction in Type II error is required, one can either raise the whole number of presentations or increase $t_{0}$. For instance, the power of the test increases to $90 \%$ when the whole number of presentations becomes 100 (see Table 12). If, in addition, the range of admissible threshold fluctuations $t_{0}$ is widened to $4 \mathrm{~dB}$, the power increases to $99 \%$ (Table 13).

\section{Appendix 1}

Since Fisher's approach implies an evaluation of the exact probability distribution of the experimental outcome for every $z_{i}$, let us evaluate the probability of observing the table $T^{(i)}$

Table 11 Relative frequencies of false rejections of the null hypothesis depending on the nominal level of significance $\alpha$

\begin{tabular}{lllllll}
\hline & $\alpha=0.01$ & $\alpha=0.05$ & $\alpha=0.1$ & $\alpha=0.15$ & $\alpha=0.2$ & $\alpha=0.25$ \\
\hline$m=5 ; k=5$ & 0.0062 & 0.0391 & 0.0858 & 0.1259 & 0.1825 & 0.2201 \\
$m=5 ; k=10$ & 0.0087 & 0.0495 & 0.1001 & 0.1511 & 0.2031 & 0.2504 \\
$m=10 ; k=5$ & 0.0085 & 0.0496 & 0.0942 & 0.1446 & 0.1972 & 0.2434 \\
$m=5 ; k=20$ & 0.0074 & 0.0501 & 0.0992 & 0.1452 & 0.1946 & 0.2473 \\
\hline
\end{tabular}

Table 12 The power of the test for the shift between two psychometric functions $t_{0}=2 d B$

\begin{tabular}{lllllll}
\hline & $\alpha=0.01$ & $\alpha=0.05$ & $\alpha=0.1$ & $\alpha=0.15$ & $\alpha=0.2$ & $\alpha=0.25$ \\
\hline$m=5 ; k=5$ & 0.0612 & 0.2027 & 0.3366 & 0.4240 & 0.5198 & 0.5813 \\
$m=5 ; k=10$ & 0.1564 & 0.3675 & 0.5210 & 0.6213 & 0.6972 & 0.7543 \\
$m=10 ; k=5$ & 0.1556 & 0.3809 & 0.5293 & 0.6302 & 0.7021 & 0.7589 \\
$m=5 ; k=20$ & 0.3233 & 0.5968 & 0.7380 & 0.8156 & 0.8654 & 0.8984
\end{tabular}

(see Table 1) associated with given $z_{i}$. Since $T^{(i)}$ is specified by two independent binomial random variables, $n_{11}^{(i)}$ and $n_{21}^{(i)}$, according to the multiplication law for independent events, the probability of observing the table $T^{(i)}$ is equal to the probability of $n_{11}^{(i)}$ successes in $n_{1+}^{(i)}$ binomial trials, with probability $p_{1}^{(i)}$ of success, times the probability of $n_{21}^{(i)}$ successes in $n_{2+}^{(i)}$ binomial trials, with probability $p_{2}^{(i)}$ of success:

$$
\begin{aligned}
P\left(T^{(i)}\right)= & \left(\begin{array}{c}
n_{1+} \\
n_{11}
\end{array}\right)\left(p_{1}^{(i)}\right)^{n_{11}}\left(1-p_{1}^{(i)}\right)^{n_{12}}\left(\begin{array}{c}
n_{2+} \\
n_{21}
\end{array}\right) \\
& \times\left(p_{2}^{(i)}\right)^{n_{21}}\left(1-p_{2}^{(i)}\right)^{n_{22}} .
\end{aligned}
$$

Since the unknown parameters $p_{1}^{(i)}$ and $p_{2}^{(i)}$ are involved in Eq. 13, it cannot be evaluated. Nevertheless, as Fisher showed under the homogeneity hypothesis, it can be done on the condition of given marginal frequencies $n_{+1}^{(i)}$ and $n_{+2}^{(i)}$. Generally, being total number of yes and no responses in both sessions, these frequencies are the outcome of the experiment; so they are considered as random variables. To evaluate the conditional probability of observing the table $T^{(i)}$ with given marginal frequencies $n_{+1}^{(i)}$ and $n_{+2}^{(i)}$, we have to divide Eq. 13 by the probability of these frequencies themselves, $P\left(n_{+1}^{(i)}, n_{+2}^{(i)}\right)$ :

$$
\begin{aligned}
P\left(T^{(i)} \mid n_{+1}, n_{+2}\right) & \\
= & \frac{\left(\begin{array}{l}
n_{1+} \\
n_{11}
\end{array}\right)\left(p_{1}^{(i)}\right)^{n_{11}}\left(1-p_{1}^{(i)}\right)^{n_{12}}\left(\begin{array}{l}
n_{2+} \\
n_{21}
\end{array}\right)\left(p_{2}^{(i)}\right)^{n_{21}}\left(1-p_{2}^{(i)}\right)^{n_{22}}}{P\left(n_{+1}, n_{+2}\right)} .
\end{aligned}
$$

Table 13 The power of the test for the shift between two psychometric functions $t_{0}=4 d B$

\begin{tabular}{lllllll}
\hline & $\alpha=0.01$ & $\alpha=0.05$ & $\alpha=0.1$ & $\alpha=0.15$ & $\alpha=0.2$ & $\alpha=0.25$ \\
\hline$m=5 ; k=5$ & 0.1868 & 0.4157 & 0.5705 & 0.6622 & 0.7407 & 0.7875 \\
$m=5 ; k=10$ & 0.3795 & 0.6613 & 0.7944 & 0.8615 & 0.9032 & 0.9325 \\
$m=10 ; k=5$ & 0.3946 & 0.6858 & 0.8075 & 0.8686 & 0.9092 & 0.9339 \\
$m=5 ; k=20$ & 0.6895 & 0.9009 & 0.7380 & 0.9571 & 0.9763 & 0.9855 \\
\hline
\end{tabular}


The denominator in Eq. 14 is evaluated as the following:

$$
\begin{aligned}
& P\left(n_{+1}, n_{+2}\right)=\sum_{u+v=n_{+1}} P\left(n_{11}=u\right) P\left(n_{21}=v\right)= \\
& \sum_{u+v=n_{+1}}\left(\begin{array}{l}
n_{1+} \\
u
\end{array}\right)\left(p_{1}^{(i)}\right)^{u}\left(1-p_{1}^{(i)}\right)^{n_{1+}-u}\left(\begin{array}{l}
n_{2+} \\
v
\end{array}\right)\left(p_{2}^{(i)}\right)\left(1-p_{2}^{(i)}\right)^{n_{2+}-v} .
\end{aligned}
$$

On the hypothesis of homogeneity for one stimulus value $z_{i}, H_{0}^{(i)}: p_{1}^{(i)}=p_{2}^{(i)}=p$, the unknown parameter can be excluded from Eq. 14, thus reducing it to a simpler form:

$P\left(T^{(i)} \mid n_{+1}, n_{+2}\right)=\frac{\left(\begin{array}{c}n_{1+} \\ n_{11}\end{array}\right)\left(\begin{array}{l}n_{2+} \\ n_{21}\end{array}\right)}{\left(\begin{array}{l}n_{++} \\ n_{+1}\end{array}\right)}$.

Now that the probability (Eq. 15) depends only on observed frequencies, it can be evaluated. Note, then, that given a set of marginal frequencies, the value for one cell in the tablet $T^{(i)}$ (say, $n_{11}^{(i)}$ ) determines the other three frequencies - that is, $n_{12}^{(i)}, n_{21}^{(i)}$, and $n_{22}^{(i)}$. So, under the hypothesis of homogeneity for one stimulus value $z_{i}$, the conditional probability (16) of obtaining any particular arrangement for all four frequencies in the tablet $T^{(i)}$ can be expressed in terms of only one variable. Let us designate this variable $x_{11}^{(i)}$. It takes on the same values as $n_{11}^{(i)}$. So, under the hypothesis $H_{0}^{(i)}$, the distribution $f^{(i)}\left(x_{11}^{(i)}\right)$ conditioned by marginal frequencies is free of the unknown probability $p$ and is expressed as the hypergeometric distribution (cf. Kendall \& Stuart, 1979, p. 581):

$f^{(i)}\left(x_{11}^{(i)}\right)=\frac{\left(\begin{array}{c}n_{1+}^{(i)} \\ x_{11}^{(i)}\end{array}\right)\left(\begin{array}{c}n_{2+}^{(i)} \\ n_{+1}^{(i)}-x_{11}^{(i)}\end{array}\right)}{\left(\begin{array}{c}n_{++}^{(i)} \\ n_{+1}^{(i)}\end{array}\right)}$.

\section{Appendix 2}

Recall the definition of the convolution of two discrete probability distributions - say, $A$ and $B$ - that are generated by two independent random variables $X_{1}$ and $X_{2}$, respectively. ${ }^{10}$ Let the variable $X_{1}$ take on the values $\left(\alpha_{1}, \alpha_{2}, \ldots\right.$, $\left.\alpha_{n}\right)$, and the variable $X_{2}$ takes on the values $\left(\beta_{1}, \beta_{2}, \ldots, \beta_{m}\right)$. Let $p\left(\alpha_{i}\right)$ stand for the probability that the variable $X_{1}$ takes the value $\alpha_{i}$-that is, $p\left(\alpha_{i}\right)=P\left\{X_{1}=\alpha_{i}\right\}$. Respectively, let $p\left(\beta_{j}\right)=P\left\{X_{2}=\beta_{j}\right\}$.

\footnotetext{
${ }^{10}$ For a discussion on the convolution of numerical sequences, see, for example, Feller (1968, pp. 266-270).
}

By definition, the convolution of $A$ and $B$ is the distribution $A^{*} B$, the domain of which is

$\left\{\gamma=\alpha_{i}+\beta_{j} \mid i=1, \ldots, n ; j=1, \ldots, m\right\}$,

and the range of which is specified by the following formula:

$P\left(\gamma_{q}\right)=\sum_{i, j: \alpha_{i}+\beta_{j}=\gamma_{q}} p\left(\alpha_{i}\right) p\left(\beta_{j}\right)$.

The probability distributions of $v_{U}^{(i)}$ and $v_{L}^{(i)}$ are given by Tables 2 and 3. Sequentially applying this formula to $m$ discrete distributions $A_{U}^{(i)}$ (so that $A_{U}^{(i)}$ is convoluted with $A_{U}^{(2)}$, and the resultant $A_{U}^{(1)} * A_{U}^{(2)}$ convoluted with $A_{U}^{(3)}$, and so on), and similarly to all $A_{L}^{(i)}$, we get two resultant discrete distributions for $v_{U}$ and $v_{L}$, respectively. Let us designate them $\left\{P_{U}\left(\zeta_{1}\right), \ldots, P_{U}\left(\zeta_{Q}\right)\right\}$ and $\left\{P_{L}\left(\zeta_{1}\right), \ldots, P_{L}\left(\zeta_{Q}\right)\right\}$. Now $p$-values for the observed $v_{U}$ and $v_{L}$ are evaluated as

$P_{U}=\sum_{i: \zeta \leq v_{U}} P_{U}\left(\zeta_{i}\right)$ and $P_{L}=\sum_{i: \zeta \leq v_{L}} P_{L}\left(\zeta_{i}\right)$.

(A program to perform the calculations of the discrete convolution, written in $\mathrm{C}++$, is available on request from the authors.)

\section{Appendix 3}

For the sake of simplicity, let us consider first the case where a random variable $\zeta$ has the continuous cumulative distribution function $F_{(x)}=P\{\zeta \leq x\}$. In this case, it can be easily proved that a random variable $F(\zeta)$ is uniformly distributed over the interval [0;1]. Indeed, let us evaluate the cumulative distribution function of $F(\zeta)$. Given $0 \leq z \leq$ 1, we have the following sequence of obvious equations: $P\left\{F_{(\zeta)} \leq z\right\}=P\left\{\zeta \leq F^{-1}(z)\right\}=F\left(F^{-1}(z)\right)=z$. Therefore, the graph of the cumulative distribution function for $F(\zeta)$ is linear on the interval $[0 ; 1]$.

Because of discreteness, the $p$-values in question are distributed only approximately uniformly, and, thus, their cumulative distribution functions are only approximately linear (as in Fig. 3).

\section{References}

Agresti, A. (1984). Analysis of ordinal categorical data. New York: Wiley.

Agresti, A. (1990). Categorical Data Analysis. New York: John Wiley and Sons.

Barlow, R. E., Bartholomew, D. J., Bremmer, J. M., \& Brunk, H. D. (1972). Statistical inference under order restrictions: 
The theory and application of isotonic regression. London: Wiley.

Berkson, J. (1978). In dispraise of the exact test. Do the marginal totals of the $2 \times 2$ table contain relevant information respecting the table proportions. Journal of Statistical Planning and Inference, 2, 27-42.

Camilli, G. (1990). The test of homogeneity for $2 \times 2$ contingency tables: A review and some personal opinions on the controversy. Psychological Bulletin, 108, 135-145.

Everitt, B. (1992). The analysis of contingency tables (2nd ed.). London: Chapman and Hall.

Everitt, B. (1993). Some aspects of the analysis of categorical data. In C. Karen \& C. Lewis (Eds.), A handbook for data analysis in the behavioral sciences (Vol. 1, pp. 321-346). Hillsdale, NJ: Erlbaum.

Feller, W. (1968). An introduction to probability theory and its applications, vol. 1 (3rd ed.). New York: Wiley.

Guilford, J. (1954). Psychometric methods (3rd ed.). New York: McGraw-Hill.

Haber, M. (1986). An exact unconditional test for the $2 \times 2$ comparative trial. Psychological Bulletin, 99, 129-132.

Haber, M. (1990). Comments on "The test of homegeneity for $2 \times 2$ contingency tables: A review and some personal opinions on the controversy" by G. Camilli. Psychological Bulletin, 108, 146-149.

Kendall, M. \& Stuart, A. (1979). The Advanced Theory of Statistics, vol. 2 (4th ed.). Wycombe: Griffin and Co. Ltd.
Klein, S. A. (2001). Measuring, estimating, and understanding the psychometric function: A commentary. Perception and Psychophysics, 63, 1421-1455.

Kroll, N. (1989). Testing independence in a $2 \times 2$ contingency table. Journal of Educational Statistics, 14, 47-79.

Mehta, C. R. (1994). The exact analysis of contingency tables in medical research. Statistical Methods in Medical Research, 3 $135-156$.

Nachmias, J. (1981). On the psychometric function for contrast detection. Vision Research, 21, 215-223.

Robertson, T., Wright, F., \& Dykstra, R. L. (1988). Order restriced statistical inference. Chichester, U.K.: Wiley.

Rosenthal, R. (1993). Cumulating evidence. In C. Karen \& C. Lewis (Eds.), Data analysis in the behavioral sciences: Methodological issues (pp. 519-559). Hillsdale, NJ: Erlbaum.

Wichmann, F. A. \& Hill, N. J. (2001a). Psychometric function: I. Fitting, sampling, and goodness of fit. Perception \& Psychophysics 63, 1293-1313.

Wichmann, F. A. \& Hill, N. J. (2001b). Psychometric function: II. Bootstrap-based confidence intervals and sampling. Perception \& Psychophysics, 63, 1314-1329.

Woodworth, R., \& Schlosberg, H. (1955). Experimental psychology (3rd ed.). London: Methuen.

Zelen, M. (1971). The analysis of several $2 \times 2$ contingency tables. Biometrica, 58, 129-137. 